\title{
Lorna Milne, Patrick Chamoiseau. Espaces d'une écriture antillaise
}

\section{Alessandro Corio}

\section{(2) OpenEdition}

1 Journals

\section{Edizione digitale}

URL: https://journals.openedition.org/studifrancesi/46380

DOI: 10.4000/studifrancesi.46380

ISSN: 2421-5856

\section{Editore}

Rosenberg \& Sellier

\section{Edizione cartacea}

Data di pubblicazione: 1 octobre 2007

Paginazione: 486-487

ISSN: 0039-2944

\section{Notizia bibliografica digitale}

Alessandro Corio, «Lorna Milne, Patrick Chamoiseau. Espaces d'une écriture antillaise», Studi Francesi [Online], 152 (LI | II) | 2007, online dal 30 novembre 2015, consultato il 24 novembre 2021. URL: http:// journals.openedition.org/studifrancesi/46380 ; DOI: https://doi.org/10.4000/studifrancesi.46380

Questo documento è stato generato automaticamente il 24 novembre 2021.

\section{(c) (i) (9)}

Studi Francesi è distribuita con Licenza Creative Commons Attribuzione - Non commerciale - Non opere derivate 4.0 Internazionale. 


\title{
Lorna Milne, Patrick Chamoiseau. Espaces d'une écriture antillaise
}

\author{
Alessandro Corio
}

\section{NOTIZIA}

LORNA MILNE, Patrick Chamoiseau. Espaces d'une écriture antillaise, Amsterdam - New York, Rodopi, «Francopoliphonies», 2006, pp. 226.

Il tema dello spazio e della sua rappresentazione è centrale nelle letterature postcoloniali, e in particolare nelle letterature caraibiche. Lo dimostra ampiamente questo lavoro di Lorna Milne sulla rappresentazione dello spazio nella scrittura di Patrick Chamoiseau. La studiosa è, tuttavia, ben consapevole dei limiti e dei rischi che un'analisi incentrata sullo spazio può comportare, sia dal punto di vista letterario che storico-antropologico. Il rischio maggiore è senz'altro quello di cadere in una sorta di “determinismo paesaggistico", per cui l'identità culturale di un popolo, tanto quanto le caratteristiche specifiche di una scrittura, risultino determinate dal territorio di appartenenza, spingendo così a una riformulazione identitaria in termini di radicamento e di appartenenza spaziali. Questo rischio è però subito scansato dalla critica scozzese, che fissa, sin dall'introduzione, una serie di riferimenti culturali e metodologici importanti che guideranno il suo lavoro. Si tratta, innanzitutto, di mettere a fuoco il legame simbiotico e ambivalente tra le specificità concrete di uno spazio e le particolarità della cultura che lo abita, e, soprattutto, la loro reciproca integrazione per la costruzione di un'identità collettiva. Risulta quindi particolarmente significativa la citazione di uno studio sul paesaggio e la memoria dello storico Simon Schama: «Les paysages sont culturels avant d'être naturels: ce sont des constructions que l'imaginaire projette sur les bois, l'eau, le rocher. [...] une fois qu'une certaine idée du paysage, un mythe, une vision s'etablissent en un lieu donné, ils ont le don de brouiller les catégories, et de rendre la métaphore plus réelle que son référent [...]» (p. 15). 
2 La natura, quindi, è culturale, ancor prima di essere naturale. Questo semplice assunto risulta pertanto indispensabile per un'analisi dei sistemi simbolici, culturali, linguistici che interagiscono nella costruzione e nella ridefinizione continua dell'identità culturale, come lo hanno pienamente dimostrato gli studi di sociologi e antropologo che la Milne chiama qui a raccolta, quali Claude Lévi-Strauss, Pierre Bourdieu, Michel de Certeau e Marc Augé. Quest'ultimo, in particolare, parla di "luoghi antropologici", qualificandoli come spazi «dont l'analyse a du sens parce qu'ils ont été investis de sens». E questo, come sa bene qualsiasi lettore di scrittori caraibici, è senz'altro vero per una realtà storica e culturale come quella delle Antille, caratterizzata da un rapporto estremamente problematico dei popoli che abitano queste isole con il loro spazio. Ed è questo, inoltre, uno dei temi centrali della scrittura di Chamoiseau, che si è da sempre mostrato particolarmente sensibile ai nodi problematici posti dalla relazione con uno spazio segnato dalle strutture e dalle gerarchie della dominazione coloniale. Come i "luoghi antropologici" descritti da Augé, quindi, lo spazio antillese è investito di un "senso" che si fonda su una certa percezione del proprio passato, trattandosi quindi di luoghi identitari, relazionali e, soprattutto, storici. Spazio, tempo, memoria, identità e linguaggio: questi gli elementi fondamentali che sono in gioco nella problematica definizione del rapporto di un popolo al suo "entour". La complessa realtà storicoantropologica dei cosiddetti "popoli della Tratta" presenta una serie di cronotopi ricorrenti, soprattutto a livello letterario, la cui analisi risulta indispensabile: la "Plantation", la "Traite", il "bateau négrier", il "marronange", la produzione di "sucre" e di "rhum", ecc. Citando un importante studio di Antonio Benitez-Rojo, The Repeating Island, l'autrice sottolinea quindi due componenti essenziali, due "desideri spaziali" tra cui l'identità antillese oscilla in continuazione: la fuga e il radicamento, l'ici e il là-bas. Lo spazio antillese risulta pertanto un'espressione metaforica della propria realtà geografica, secondo quella che il critico americano Eric Sellin ha definito una "sinestesia generica", ossia «une symbiose entre les pulsions métaphoriques de l'esprit d'un auteur et les différents stimuli produits par son environnement» (p. 21). Sellin vede quindi, nella topografia e nella natura antillesi, un complesso di significazioni che permette allo scrittore di fare convergere l'espressione estetica e l'esperienza vissuta, riattivando così, anche sul piano metaforico, quell'elemento indispensabile all'identità che è la storia.

3 Ed è proprio a livello del rapporto tra spazio e storia che la produzione letteraria e teorica di Chamoiseau mette in luce quella "déresponsabilisation généralisé" come esito della frattura culturale e identitaria che contraddistingue lo spazio dominato della "départementalisation". Gli spazi letterari, nell'opera di Chamoiseau, sono infatti delle costruzioni investite di cultura, quindi di storia e di politica, in cui le specificità topografiche che caratterizzano tutta la sua produzione narrativa, da Chronique des sept misères a Biblique des derniers gestes, veicolano una carica identitaria potente e fortemente ancorata nell'ici della Martinica. La scrittura di Chamoiseau, secondo Lorna Milne, può essere definita come un «enracinement politisé dans l'espace géographique particulier des Antilles» (p. 31), radicamento caratterizzato sia dalla ri-scrittura della storia, ma anche, e soprattutto, da una re-iscrizione nel paesaggio: questo avviene soprattutto in romanzi quali Chronique des sept misères, Solibo magnifique e Texaco, in cui la finzione narrativa è inscritta in scenari storicamente e topograficamente reali e riconoscibili. Si tratta di una vera e propria presa di possesso dello spazio, di fronte ai rapporti problematici alla terra e al pays che hanno sempre caratterizzato gli scrittori e i popoli creoli. In Écrire en pays dominé, Chamoiseau ha ben analizzato, infatti, proprio 
quel passaggio da una "dominazione brutale" a una "dominazione silenziosa" operato dalla dipartimentalizzazione, sottolineando, come già aveva fatto Fanon, tra i risultati di questa subordinazione e alienazione culturale quella trasformazione mentale del colonizzato che lo spinge a integrare la "funzione colonizzatrice", a costruirsi dei masques blancs.

Lo studio di Lorna Milne si pone dunque come obiettivo primario quello di esplorare le strategie dispiegate da Chamoiseau per esprimere, interrogare, e infine superare questo rapporto difficile allo spazio del pays natal, consapevole che esplorare l'evocazione dello spazio significa anche avvicinarsi all'espressione dell'identità. Come afferma lo stesso Chamoiseau, infatti, «la problématique d'exploration identitaire est une problématique de réappropriation de l'espace, tant symboliquement que concrètement» (p. 33) e pertanto agli elementi dello spazio, della storia e dell'identità si aggiunge, nella scrittura di questo autore, la problematica del molo e dell'identità dello scrittore. Il secondo obiettivo dell'analisi della Milne sarà quindi quello di discernere, attraverso il prisma spaziale, il molo che Chamoiseau riserva allo scrittore antillese e di definire la sua "poetica dello spazio". Nei sei capitoli del libro Lorna Milne si concentrerà su quattro spazi privilegiati nell'opera narrativa di Chamoiseau, su quattro cronotopi e sulle loro potenzialità simboliche: il "bateau négrier", legato ai temi della memoria e delle origini, il "marché", legato invece all'apertura e agli scambi interculturali, l'"habitat créole", caratterizzato dalla fragilità delle costruzioni sociali, e infine il "bois", legato ai temi della ricerca, della trasformazione politica e della metamorfosi letteraria, per concludere infine sulla poetica del déplacement, dello spaesamento e della meraviglia, anch'essi elementi ricorrenti nell'opera dello scrittore martinicano. Si tratta, quindi, di uno studio ricco, metodologicamente ben impostato e che prende in conto meticolosamente tutta l'opera di Chamoiseau, film compresi, fornendo inoltre, strumento prezioso per i suoi lettori, un breve glossario di termini creoli ricorrenti nei suoi romanzi e racconti. 